\title{
Knee Infection Caused by Candida Albicans Following a Knee Arthroscopy - A Case Report
}

\author{
Ares $\mathrm{O}^{1,2,3^{*}}$, Seijas $\mathrm{R}^{2}$, Albareda $\mathrm{D}^{4}$ and Sallent $\mathrm{A}^{5}$ \\ ${ }^{1}$ Hospital Clinic Barcelona, Barcelona, Spain \\ ${ }^{2}$ Fundación García-Cugat, Hospital Quirón, Barcelona, Spain \\ ${ }^{3}$ International University of Catalonia, Spain \\ ${ }^{4}$ Hospital Viladecans, Barcelona, Spain \\ ${ }^{5}$ Hospital Vall d'Hebron, Barcelona, Spain
}

*Corresponding author: Ares O, MD, PhD Orthopedic Surgery Department, Hospital Clinic BarcelonaVillarroel, 170, 08036 Barcelona, Spain, Tel: +34 932275400, Fax: +34 932022347, E-mail: arestraumatologia@gmail.com

Citation: Ares O, Seijas R, Albareda D, Sallent A (2014) Knee Infection Caused By Candida Albicans Following a Knee Arthroscopy - A Case Report. SAJ Cas Rep 1: 203. doi: 10.18875/2375-7043.1.203

Article history: Received: 03 September 2014, Accepted: 04 November 2014, Published: 06 November 2014

\begin{abstract}
Introduction: musculoskeletal infection by Candida albicans is a rare condition, even more following a knee arthroscopy as the present case report.

Case presentation: we present a non-immunosuppressed patient with a bad post-operative knee arthroscopy who developed infection by Candida albicans. Candida infections are rare in the musculoskeletal system, except for immunosuppressed patients. After presenting with high fever and tension-effusion on his knee, cultures were found with Candida albicans. Specific antifungal treatment (according to the antifungiogram) was performed with poor clinical outcome and given the patient's poor condition several surgical debridement was required besides further antifungal treatment. Final treatment for its resolution was combining an antifungal treatment and a knee arthrodesis.
\end{abstract}

Conclusion: infection by Candida albicans is a highly dangerous condition for the joint's viability that must be closely monitored.

Keywords: Candida albicans; Arthroscopy; Knee; Surgical Site Infection

\section{Introduction}

Candidiasis is a disease caused by the infection of a fungus, Candida albicans, which is more commonly observed at the skin, oral and genital mucosa, and less frequently (and secondary to its dissemination through blood stream) it can compromise other organs such as kidney, liver, heart, meninges, and bone in the form of osteomyelitis, generally in immunodeficient patients, diabetic, or those with associated severe pathologies, cancer or having been hospitalized in intensive care units. Musculoskeletal infection is a rare condition [1-4], and even more if it is secondary to a knee arthroscopy.

\section{Case report}

We present an 85 year-old male, with no relevant past medical history other than benign prostatic hyperplasia, with no infectionrisks associated.

The patient presented to our clinic complaining of unstable knee. Physical examination revealed a torn external meniscus in his right knee (McMurray test positive), diagnosis confirmed with MRI. A conventional arthroscopy was performed, with the common two portals and 45 minutes-duration of the leg's ischemia. During surgery, an external meniscectomy was performed with no associated complications (Figure 1). Postoperatively, patient presented an intermittent clinical manifestation of urinary retention, which was resolved with a urinary catheter. The initial progress of the knee was correct. 15 days postoperative, the patient readmits due to high fever $\left(38.8^{\circ} \mathrm{C}\right)$, associated with synovial tension-effusion of his right knee. An arthrocentesis is performed in order to release pain and for further diagnosis, and blood analysis as well as cultures of the synovial liquid are run. No other site of 
infection is detected. Blood analysis shows anemia manifestations as well as acute renal failure, which associated to the infection of the surgical wound advise of the need to readmit the patient for further study and an adequate treatment.

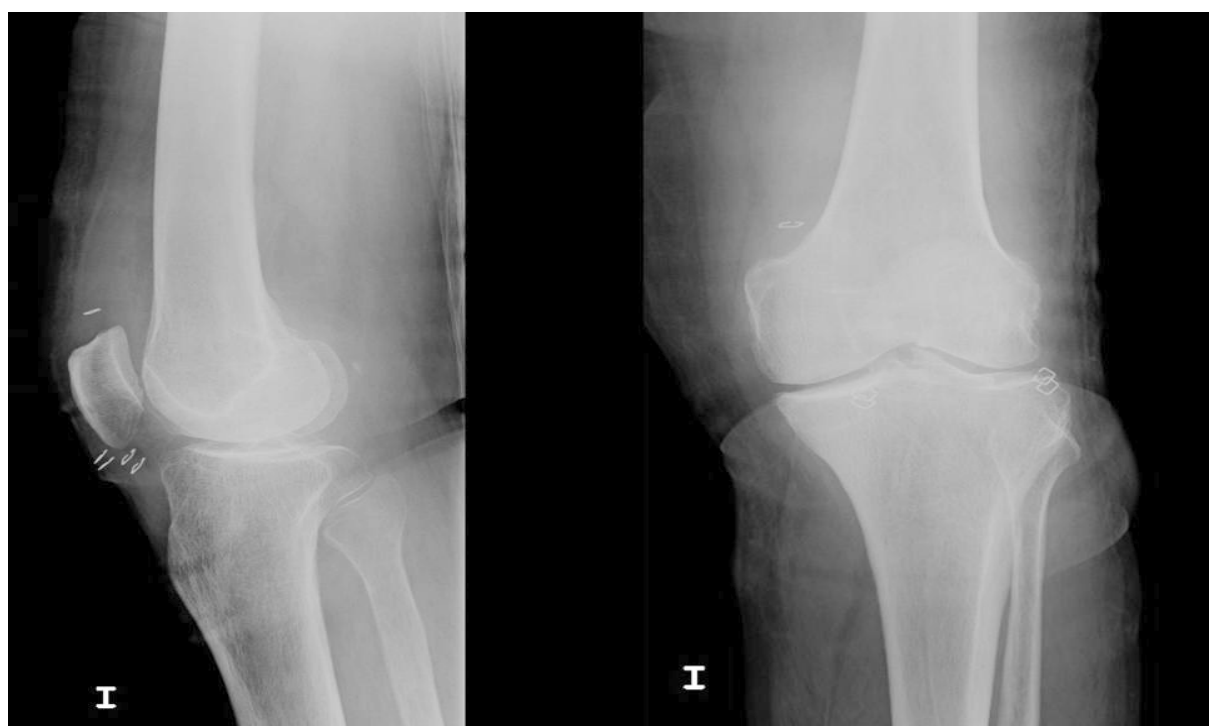

Figure 1: Lateral and anteroposterior X-ray view of the knee post-arthroscopy

The study of the synovial liquid suggests infection but does not detect bacterial flora, yet it does show growth of Candida albicans and the fungiogram describes the fungus to be fluconazole-sensible. In view of these findings, antibiotic treatment is prescribed with ceftriaxone, gentamicin and fluconazole. A surgical arthroscopic debridement is performed, during which an articular wash and sampling for biopsy and culture is carried out, demonstrating yeast growing once more. Fluconazole treatment is continued, associated to a management of the clinical manifestations of the acute renal failure originated post-renal, due to benign prostatic hyperplasia, as well as the persistent anemia.

The patient refers after intrahospitalary rehabilitation severe shortness of breath. An echocardiography discarded a heart infection and the diagnosis orientation at this point was of pulmonary thromboembolism, confirmed by a thoracic angio-CT, introducing immediate anticoagulant therapy. A new arthroscopy with a new debridement was performed and the persistence of Candida albicans infection was confirmed. The patient continued with pain symptoms and articular liquid effusion, reason for what we decided to replace fluconazole with caspofungina. An immunosuppression study was carried out which, although its nonconclusive result, suggested myeloma or paraproteinemia.

Given the poor clinical progress of the knee, several joint lavages were required. An indium-111 leukocyte bone scan was performed confirming right septic arthritis. After 5 weeks and given the absence of clinical results, the treatment was changed to Voriconazol, with which the patient improved and thus we decided his discharge.

During the following three months he continued with Voriconazol PO and with no symptoms of knee inflammation, but yet with persistent anemia and one gastroenteritis episode. However, after four months the patient presented once more knee pain at standing. A new MRI was performed, showing an extensive osteomyelitis in femur, tibia and patella with feasible abscess. Given the results, the patient was hospitalized for a surgical debridement and a new prolonged antifungal treatment. During the surgical procedure, a bone biopsy was obtained, which confirmed the persistence of a fungal infection together with destruction of the knee joint and the surroundings bones.

A new bone scan confirmed the diagnosis of femoral and tibial osteomyelitis and we decided to perform a new surgical procedure due to the above-mentioned biopsy encounters. We performed an arthrodesis removing articular surfaces and placing an external fixation (Ilizarov type) for six months (Figure 2). On the whole, the patient underwent this surgery after 7 months of the primary surgery. During postoperative period he presented an abscess at one of the needles that required drainage and replacement. An infection by Staphylococcus aureus was documented and starting antibiotics to treat this infection.

The patient has not presented infection recurrence since the arthrodesis two years ago, and the imaging shows bone consolidation in the knee with a $6 \mathrm{~cm}$ dysmetria.

\section{Discussion}

The incidence of knee infection after arthroscopy is very low (0.04-0.1\%) [5,6], and even less common is the infection by Candida albicans in the musculoskeletal system. The patient probably became contaminated during the procedure or got a fungemia that compromised the operated knee [4]. No immunosuppression was documented on the patient, besides his elderly age. 


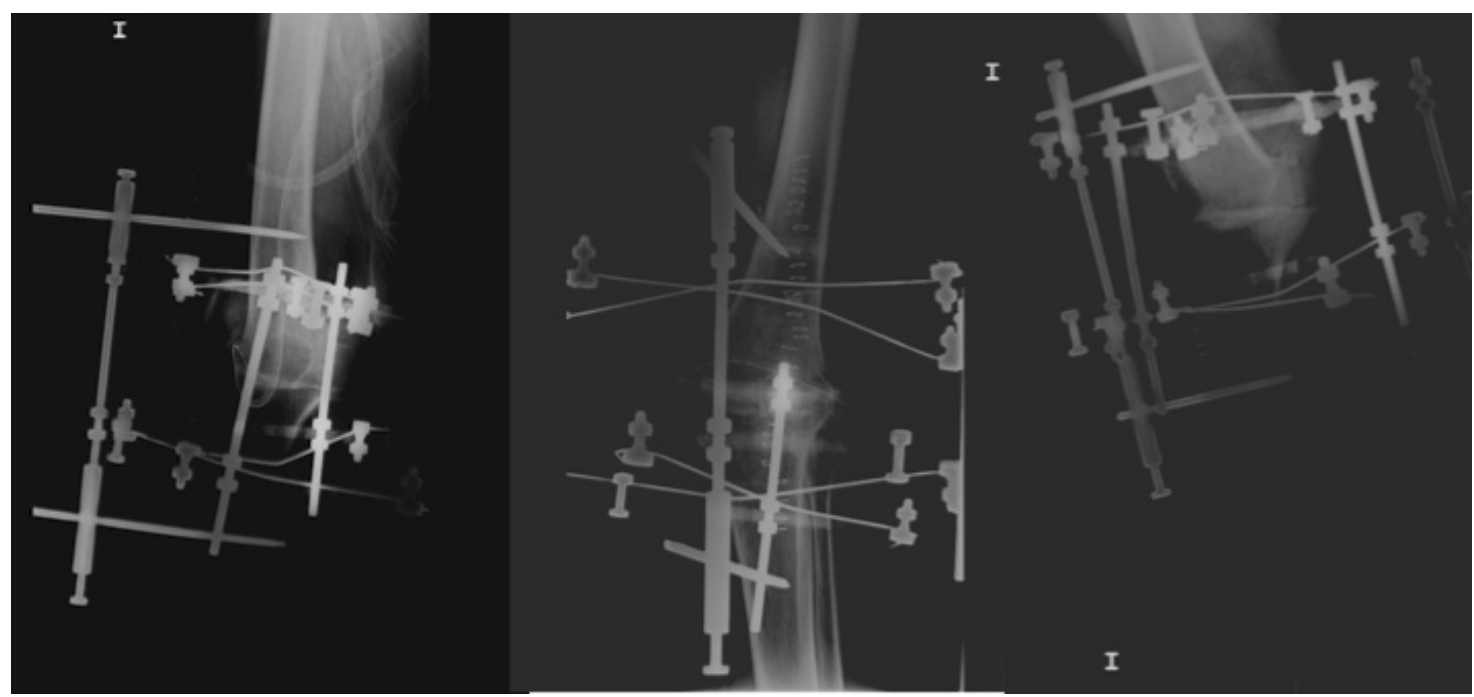

Figure 2: From left to right, three X-ray images from the knee arthrodesis; lateral, anteroposterior and lateral

The diagnosis, which in a beginning was not complex although it appeared with the same symptoms as a bacterial infection, turned out to have a complicated progress due to the failure of the first prescribed antifungal drugs, which were selected by the antifungiogram. To make matters worse, the patient had prostatic and renal pathologies associated, together with a pulmonary thromboembolism. Following the first surgery, the patient required a urinary catheter for acute urinary retention, which could be the origin of infection, however, the patient did not present urinary tract symptoms. Finally, the patient improved with the third antifungal, Voriconazol [7], but after ceasing the treatment he presented symptomatology recurrence together with severe articular involvement, as a result of which the patient underwent surgery [8].

Of all the published rare infections in the musculoskeletal system, as Pseudomonas aeruginosa [3], Clostridium perfringens [1], Neisseria meningitides [2] and Candida albicans [4], all of them had successful outcomes except for Candida albicans, which required articular arthrodesis, just as in our case.

The different possibilities of treatment when facing articular infection include arthroscopic debridement, open surgery (arthrotomy) and suction-puncture several times. When possible, the treatment to choose should be the less aggressive arthroscopic debridement $[9,10]$, as we did with our patient. Morbidity of arthroscopic debridement compared to suction-puncture is lower, allowing a complete synovectomy (subquadricipital cul-de-sac, lateral groove, medial groove and anterior compartment). Immediately we perform both posteromedial and posterolateral portals to conclude synovectomy and the arthroscopic debridement.

Obviously, all these treatments have to be accompanied by a correct antibiotic treatment identified with the antibiogram/ antifungiogram. The main objective is the articular decompression, in this case of the knee, withdrawal of proteolytic bacterial enzymes and of course, eradicating infection in order to avoid destruction of articular cartilage and bone infection.

\section{Conclusion}

To conclude, infection by Candida is highly dangerous condition for the joint's viability, due to its difficult management and treatment resistance. Therefore it is very important to keep a close monitoring of those patients who are diagnosed with this infection, besides a prompt diagnosis and management in order to obtain better outcomes.

\section{References}

1. Bernhang AM (1987) Clostridium pyoarthrosis following arthroscopy. Arthroscopy 3: 56-8.

2. Christopher GW, Jurik JA, Janecki CJ, Haake PW, Riley GJ, et al. (1982) Meningococcal arthritis, bacteremia, and osteomyelitis following arthroscopy. Report of a case. Clin Orthop Relat Res 171: 127-30.

3. Montgomery SC, Campbell J (1989) Septic arthritis following arthroscopy and intra-articular steroids. J Bone Joint Surg Br 71: 540.

4. Wind WM, McGrath BE, Mindell ER (2001) Infection following knee arthroscopy. Arthroscopy 17: 878-83.

5. Sherman OH, Fox JM, Snyder SJ, Del Pizzo W, Friedman MJ, et al. (1986) Arthroscopy--"no-problem surgery". An analysis of complications in two thousand six hundred and forty cases. J Bone Joint Surg Am 68: 256-65.

6. Small NC (1988) Complications in arthroscopic surgery performed by experienced arthroscopists. Arthroscopy 4: 215-21.

7. Pearson MM, Rogers PD, Cleary JD, Chapman SW (2003) Voriconazole: a new triazole antifungal agent. Ann Pharmacother 37: 420-32.

8. Bargiotas K, Wohlrab D, Sewecke JJ, Lavinge G, DeMeo PJ, et al. (2007) Arthrodesis of the knee with a long intramedullary nail following the failure of a total knee arthroplasty as the result of infection. Surgical technique. J Bone Joint Surg Am 89: 103-10.

9. Smith MJ (1986) Arthroscopic treatment of the septic knee. Arthroscopy 2: 30-4.

10. Jackson RW (1985) The septic knee--arthroscopic treatment. Arthroscopy 1: 194-7. 